\title{
Hybrid Hydrogen Fuel Cell and Photo-Voltaic System for Remote Telecommunications Applications
}

\author{
Peter R. Wilson and Reuben Wilcock
}

February 10, 2013

\begin{abstract}
Recent applications of Photovoltaics and other renewable energy sources have tended to be linked to provide energy for remote applications such as desalination, telecommunications and signage. The use of Hydrogen fuel cells has also been the subject of much research for potential traction and transportation applications. This paper describes the design and implementation of a practical power system that integrates photovoltaics, battery storage and hydrogen fuel cells for remote telecommunications systems. The paper will provide an overview of the overall system architecture, details of the hardware prototype installation and some preliminary results. A wireless telemetry system and control system was also implemented to allow optimization and evaluation of different control and energy management strategies.
\end{abstract}

\section{Introduction}

With the explosion of Telecommunications globally, it has often been the case that copper or fibre infrastructure is either lagging behind, or simply too expensive to be rolled out across rural and remote communities. This can be a particular issue in developing regions of the world such as Africa and parts of Asia. In spite of this, there has been a huge demand for personal, mobile communications via cellular telephones and to use this as a mechanism to provide reasonable bandwidth communications for voice and 
data in these remote areas. The obvious infrastructure need is for a network of telecommunications masts to provide coverage to at least medium size locations. The fundamental problem with this is the potential lack of access to grid power, and therefore the masts need to be self sufficient for several months at least.

The power requirements for a typical rural mast will be of the order of several hundred Watts (peak), but this can be managed and will often be less than this, however an energy budget can be estimated for typical applications. Using the existing power grid has been proposed as a means of facilitating such communications [1], however if there is not grid in place, then this will clearly not be feasible. Using some form of Photovoltaic or wind generation is also possible, however this is clearly weather dependent, and also limited potentially by the cost and size constraints of a typical mast. In order to reduce the dependence on favourable irradiance or wind conditions, battery storage is often used, however this can lead to an expensive and vulnerable system. One approach to remote and rural settings is to consider a fuel cell approach, where there will be a much smaller battery store, however a hydrogen fuel cell is used for overnight operation and to provide power when there is not enough power from the renewable sources.

There has been quite a bit of research undertaken

\section{Design}

The approach for the design of the demonstrator system was to investigate a balance between the solar and fuel cell power capabilities and the storage required to provide back up capacity. Clearly it is advantageous to be able to use solar to charge the battery as much as possible, and resort to the fuel cell only when absolutely necessary to lengthen the time between refills of the Hydrogen cylinder in the remote location.

In order to investigate this, the system was designed from the outset to provide a completely configurable system, where each element could be switched in and out remotely, and also the load could be controlled to both mimic the load profile of a representative system, but also to allow the optimization of the in service usage of the system. The overall design of the demonstrator can be seen in 1 where the fuel cell, battery and controllable load were located in a remote box, and the system also consisted of a Hydrogen store (canister) on the ground and the solar panel mounted on the top of 


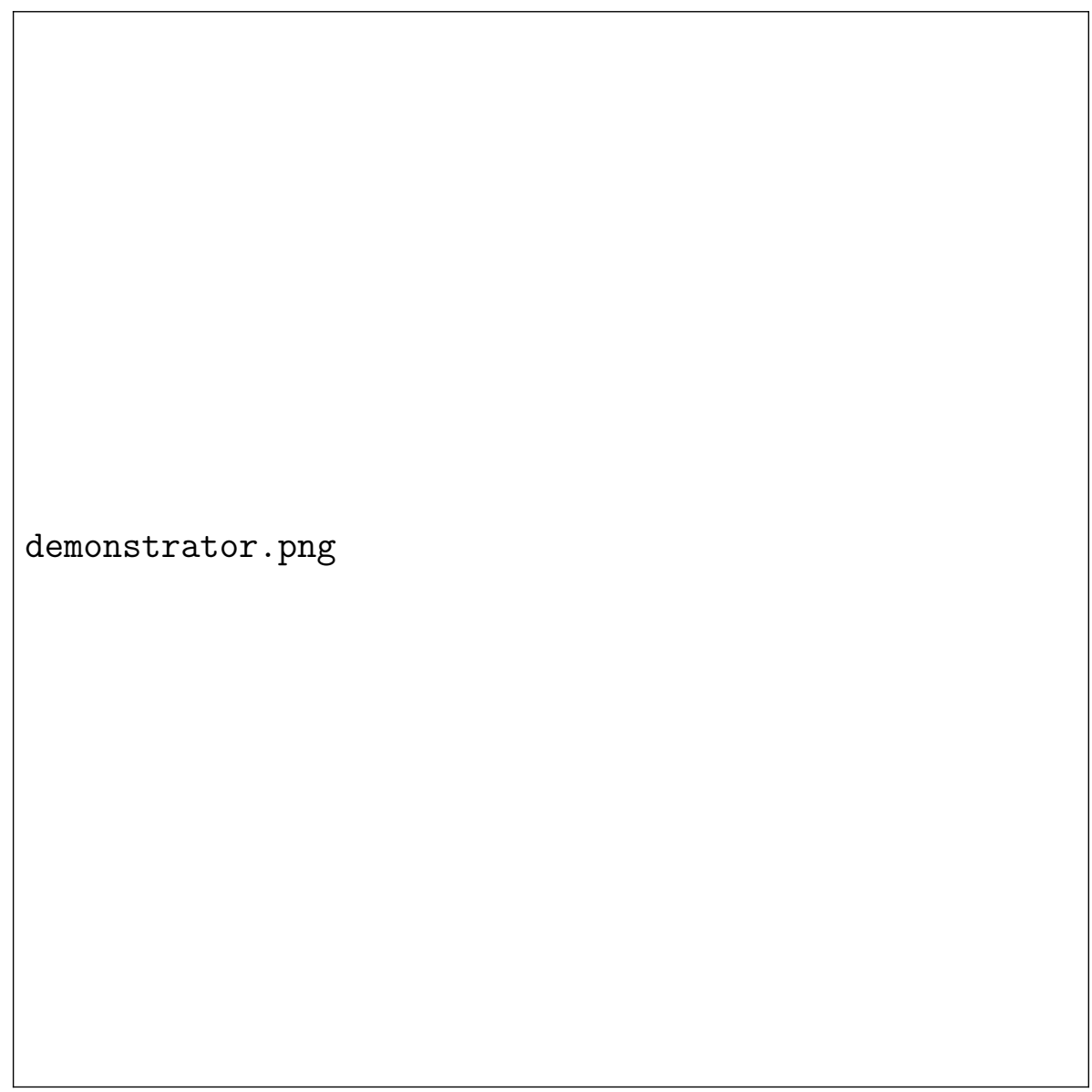

Figure 1: Demonstrator System Design 
the mast. The mast also contained a wireless radio aerial, and a monitor and control station was located in a nearby control room for remote telemetry.

\section{Implementation}

4 Results

5 Conclusions

\section{References}

[1] T. Mhlongo, T. Afullo, Power line telecommunications option in rural kwazulu-natal, in: AFRICON, 2004. 7th AFRICON Conference in Africa, Vol. 2, 2004, pp. 1263 - 1268 Vol.2. doi:10.1109/AFRICON.2004.1406895. 